\title{
Periosteal chondroma of the distal tibia: Computed tomography and magnetic resonance imaging characteristics and correlation with histological findings
}

\author{
JUN NISHIO $^{1}$, YASUHARU ARASHIRO ${ }^{1}$, SHUN MORI $^{1}$, HIROSHI IWASAKI $^{2}$ and MASATOSHI NAITO ${ }^{1}$ \\ Departments of ${ }^{1}$ Orthopaedic Surgery and ${ }^{2}$ Pathology, Faculty of Medicine, Fukuoka University, Fukuoka 814-0180, Japan
}

Received December 4, 2014; Accepted January 8, 2015

DOI: $10.3892 / \mathrm{mco} .2015 .492$

\begin{abstract}
Periosteal chondroma is a rare benign hyaline cartilage neoplasm situated on the bone surface. This is the presentation of a unique case of periosteal chondroma arising in the left distal tibial metaphysis of a 25 -year-old female patient with a history of antecedent trauma. The physical examination revealed swelling and tenderness in the anterolateral aspect of the left distal lower limb. Plain radiographs revealed a discernible soft tissue lesion with peripheral foci of mineralization. Computed tomography scans confirmed the presence of a surface-based mass with peripheral ossification and a thin rim of calcification. On magnetic resonance imaging, the well-circumscribed mass exhibited intermediate signal intensity on T1-weighted sequences and high signal intensity with foci of decreased signal intensity on T2-weighted sequences. Contrast-enhanced T1-weighted sequences revealed predominantly peripheral enhancement without intramedullary involvement. Following an open biopsy, marginal excision with curettage of the underlying bone cortex was performed. Histologically, the tumor consisted of mature hyaline cartilage arranged in distinct lobules. Foci of ossification with mature bone trabeculae forming a thin shell-like structure were identified in the periphery of the tumor. The mindbomb E3 ubiquitin protein ligase 1 labeling index was $<1 \%$. Based on these findings, the tumor was diagnosed as periosteal chondroma. There has been no evidence of local recurrence at 4 months following surgery. Despite its rarity, periosteal chondroma must be considered as a possible diagnosis when confronted with a surface-based, mineralized lesion in the metaphysis of long bones.
\end{abstract}

Correspondence to: Dr Jun Nishio, Department of Orthopaedic Surgery, Faculty of Medicine, Fukuoka University, 7-45-1 Nanakuma, Fukuoka 814-0180, Japan

E-mail: jnishio@cis.fukuoka-u.ac.jp

Key words: periosteal chondroma, tibia, computed tomography, magnetic resonance imaging, periosteal chondrosarcoma, long bones

\section{Introduction}

Periosteal chondroma, also known as juxtacortical chondroma, is a benign cartilaginous neoplasm of the bone surface that accounts for $<2 \%$ of all chondromas (1). Periosteal chondroma exhibits a notable tendency to involve the proximal humerus and distal femur. Patients present with a swelling or palpable mass that may be painful. Surgical excision is the treatment of choice. Local recurrence is extremely uncommon and is associated with incomplete excision. This is a presentation of the clinicopathological and radiological characteristics of a case of periosteal chondroma involving the distal tibia in a young adult female patient. Written informed consent was obtained from the patient for publication of this case report and accompanying images.

\section{Case report}

A 25-year-old woman was referred to our hospital for further evaluation of abnormal findings on an ankle radiograph. The patient had a history of antecedent trauma to the left distal lower limb. The physical examination revealed swelling and tenderness in the anterolateral aspect of the left distal lower limb. The laboratory data were within normal limits. The patient's medical history was non-contributory.

Plain radiographs revealed a discernible soft tissue lesion with peripheral foci of mineralization (Fig. 1). Computed tomography (CT) scans confirmed the presence of a surface-based mass with peripheral ossification and a thin rim of calcification (Fig. 2). Magnetic resonance imaging (MRI) revealed a well-circumscribed juxtacortical mass measuring $2.5 \times 1.8 \times 1.5 \mathrm{~cm}$. The mass exhibited intermediate signal intensity on T1-weighted sequences (Fig. 3A) and high signal intensity with foci of decreased signal intensity on T2-weighted sequences (Fig. 3B). Contrast-enhanced T1-weighted sequences demonstrated predominantly peripheral enhancement without intramedullary involvement (Fig. 3C). Soft tissue edema adjacent to the lesion was also observed.

Following an open biopsy, marginal excision with curettage of the underlying bone cortex was performed. Histologically, the tumor was well defined and surrounded by a periosteum-like fibrous capsule. The tumor was composed of a proliferation of chondrocytes in an abundant myxoid or chondromyxoid matrix (Fig. 4A). Foci of ossification with mature bone trabeculae 


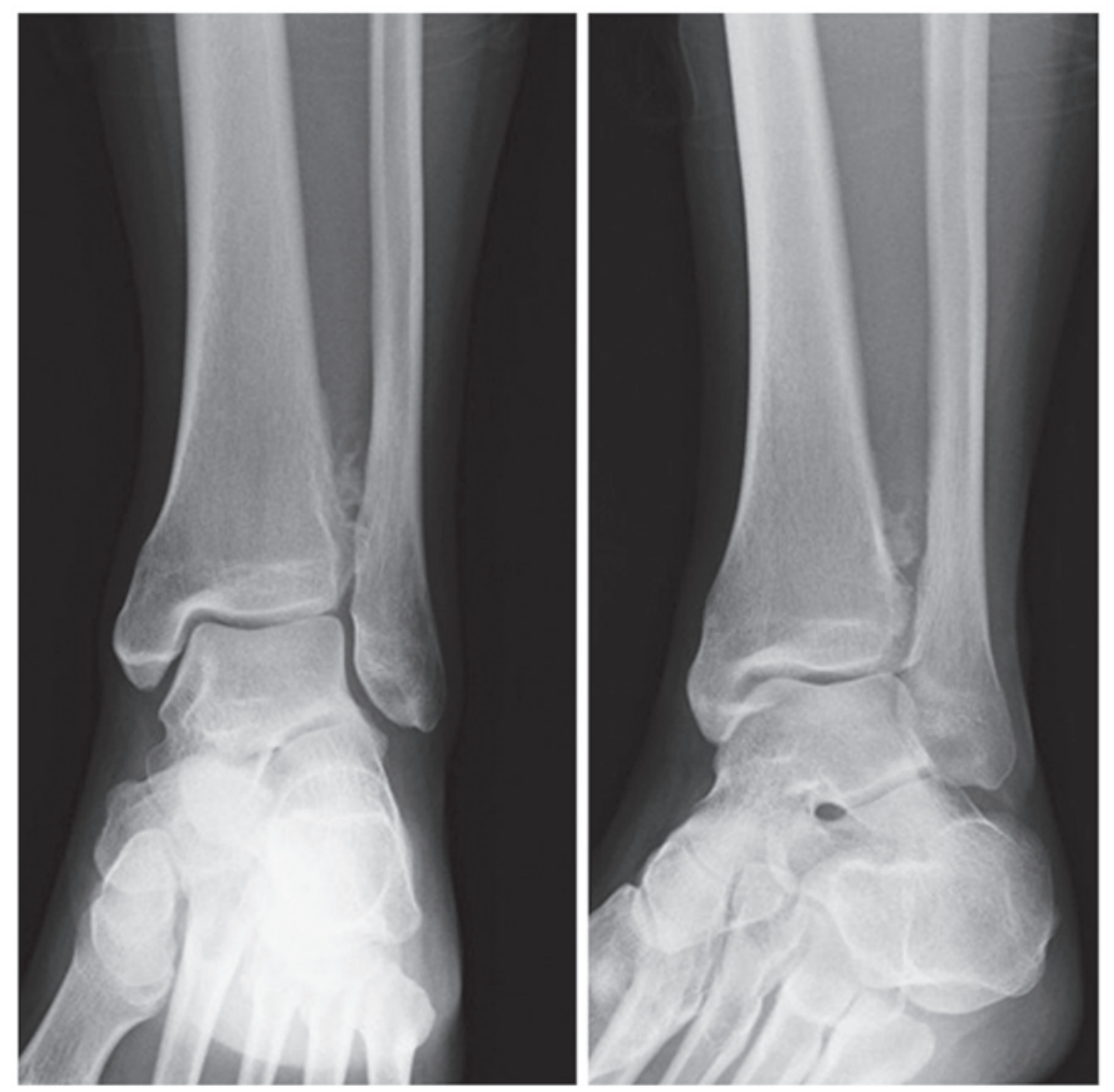

Figure 1. Plain radiographs of the left ankle revealing a discernible soft tissue lesion with peripheral foci of mineralization.
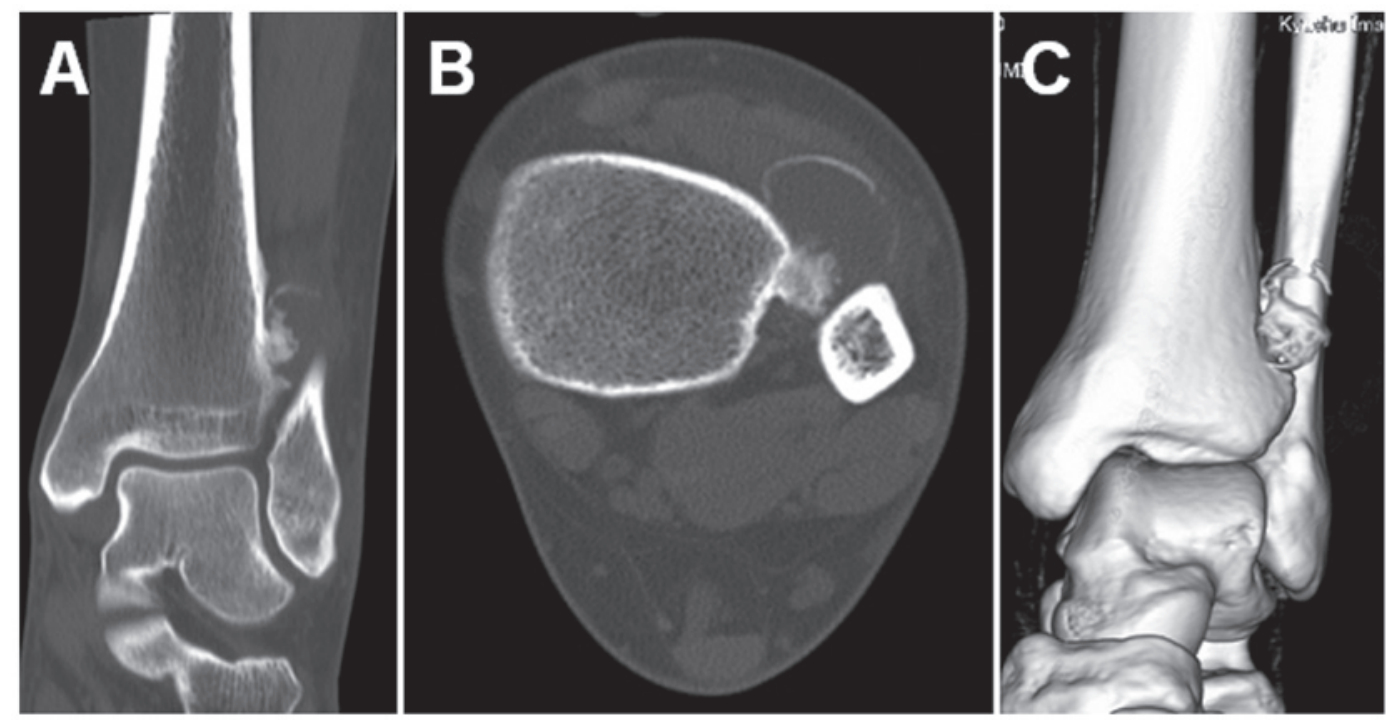

Figure 2. (A) Coronal, (B) axial and (C) three-dimensional computed tomographic imaging of the left ankle showing a surface-based mass with peripheral ossification and a thin rim of calcification.

forming a thin shell-like structure were found in the periphery of the tumor (Fig. 4B). The cytoplasm of the tumor cells was strongly positive for periodic acid-Schiff reaction. Alcian blue staining demonstrated an abundance of acid mucin in the stroma. The mindbomb E3 ubiquitin protein ligase 1 labeling index was $<1 \%$. There was no nuclear atypia and mitotic figures were not detected. Based on these characteristics, the tumor was diagnosed as periosteal chondroma.
The postoperative course was uneventful and there was no evidence of local recurrence 4 months after surgery (Fig. 5).

\section{Discussion}

Periosteal chondroma is significantly less common compared to enchondroma and predominantly occurs in children and young adults, with a marginal male predominance (1). 

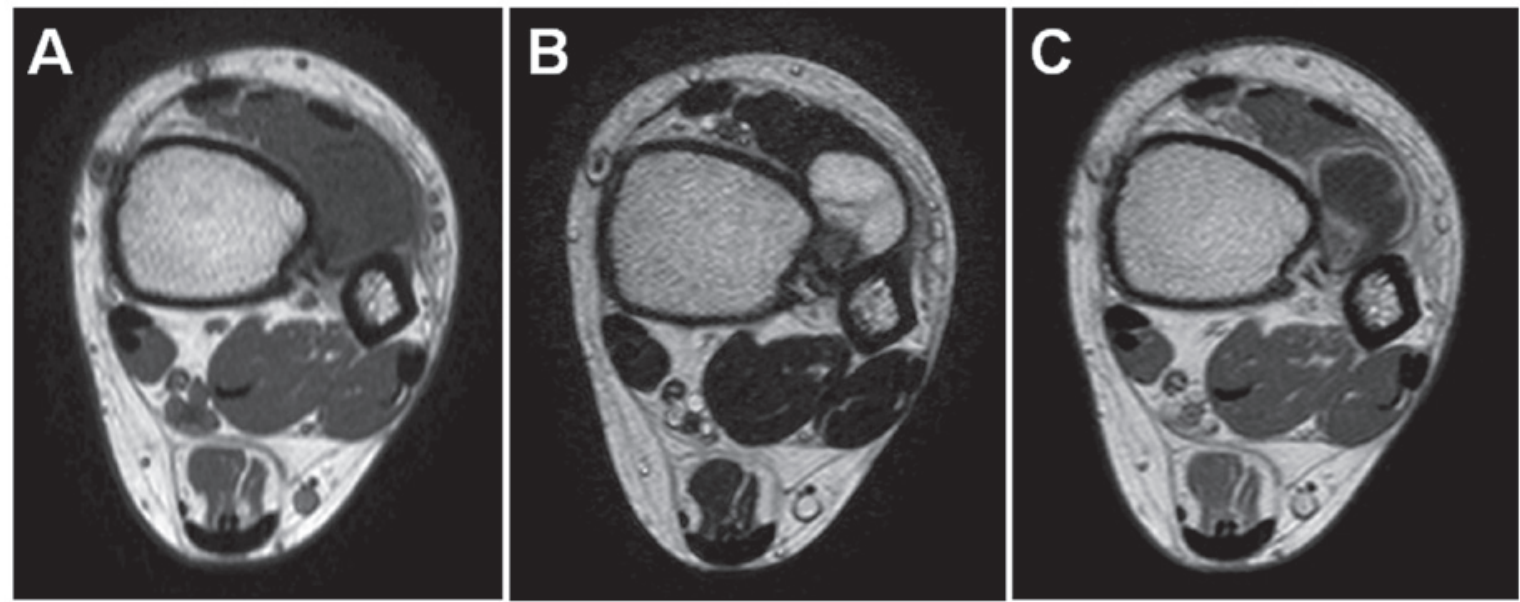

Figure 3. Axial magnetic resonance imaging showing a well-circumscribed juxtacortical mass with (A) intermediate signal intensity on T1-weighted sequence and (B) high signal intensity with foci of decreased signal intensity on T2-weighted sequence. (C) Contrast-enhanced T1-weighted sequence demonstrating predominantly peripheral enhancement. No intramedullary extension or edema was identified.
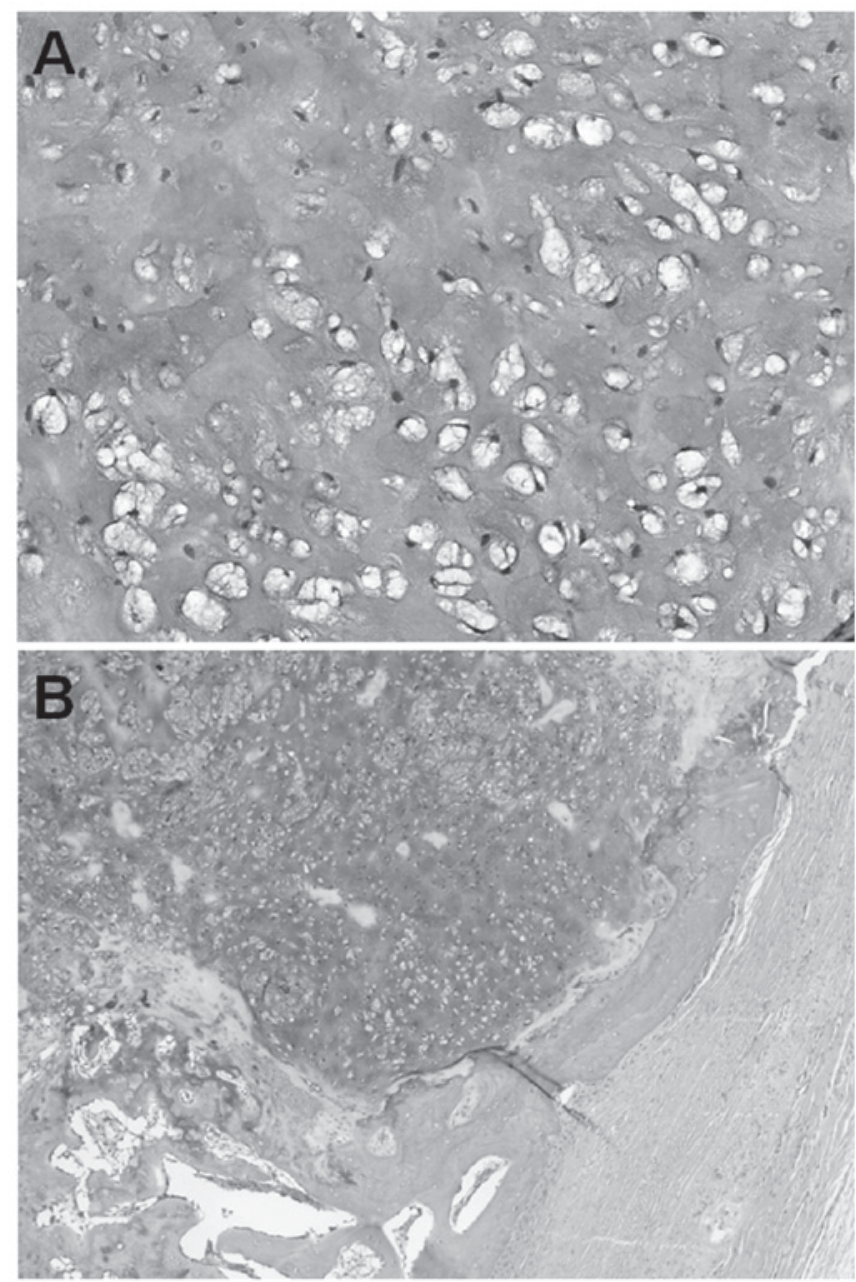

Figure 4. Histological findings of periosteal chondroma. (A) The tumor was composed of bland chondrocytes in an abundant myxoid matrix. (B) Foci of ossification with mature bone trabeculae forming a thin shell-like structure were detected in the periphery of the tumor.

Periosteal chondroma tends to arise in the metaphysis of the proximal humerus or distal femur. The small tubular bones of the hand are also common sites (2). Periosteal chondroma rarely exceeds $3 \mathrm{~cm}$ in maximum diameter and may erode the underlying cortex without penetrating into the medullar cavity.
Histologically, the lesion occasionally exhibits hypercellularity, nuclear pleomorphism and binucleation, which may lead to a misdiagnosis of chondrosarcoma. It is therefore crucial to be familiar with the imaging characteristics of periosteal chondroma, in order to avoid a misdiagnosis. 


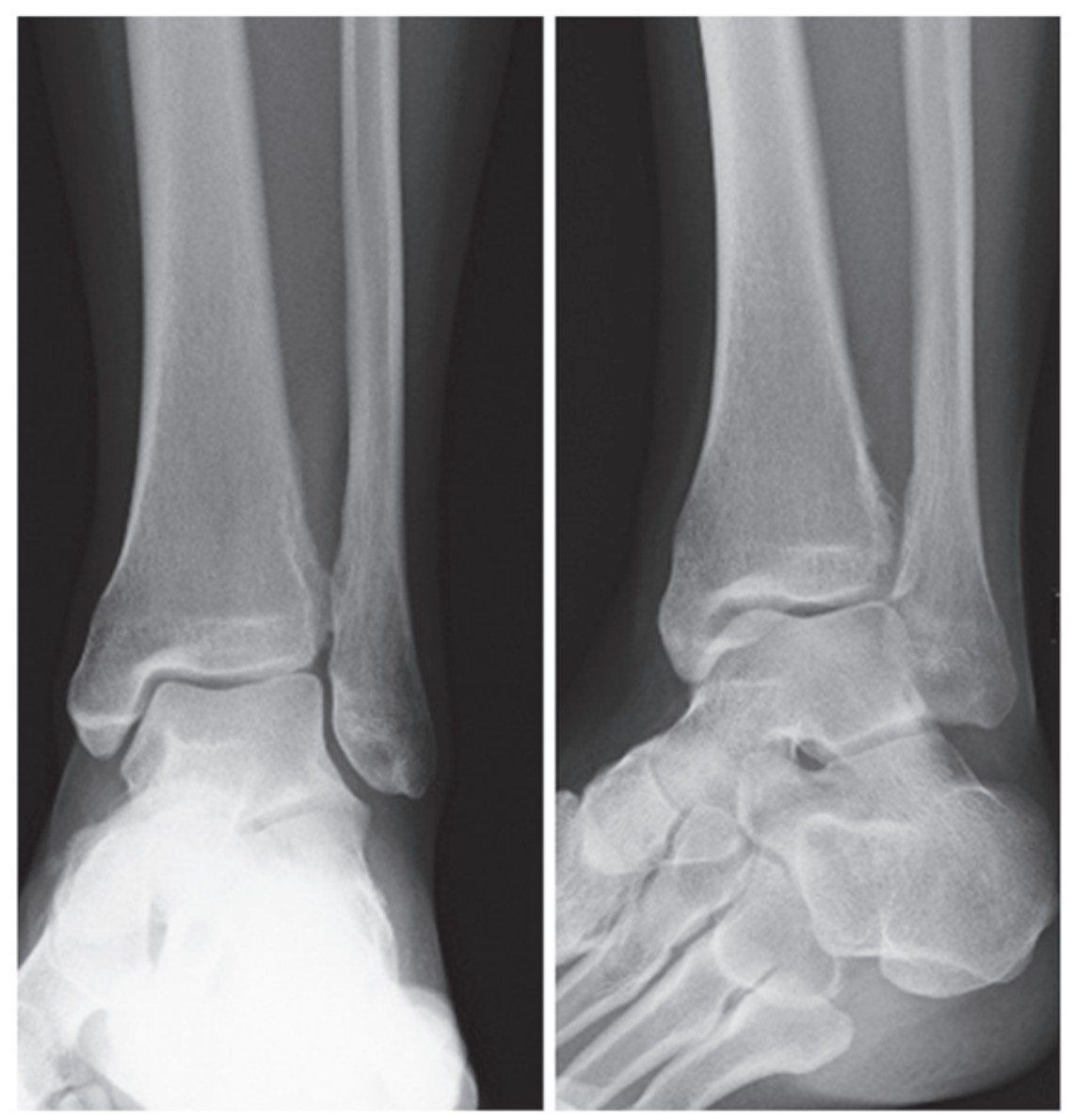

Figure 5. Follow-up plain radiographs of the left ankle 4 months after surgery.

The pathogenesis of periosteal chondroma is not well understood. It is of interest that our patient had a history of at least one traumatic event. In the present case, it was suspected that trauma may be a related or predisposing factor for the development of periosteal chondroma. However, congenital periosteal chondroma has been reported in the literature (3). Recently, heterozygous mutations of the isocitrate dehydrogenase 1 gene were detected in a number of periosteal chondromas (4).

Plain radiographs commonly reveal a discernible soft-tissue lesion with cortical scalloping, underlying cortical sclerosis and overhanging margins $(2,5)$. The lesion may exhibit a sclerotic rim or thin cortical shell. CT is useful in identifying the presence of scattered mineralizations. On MRI, periosteal chondroma typically appears as a well-circumscribed mass with intermediate signal intensity on T1-weighted sequences and high signal intensity with variable low signal intensity foci on T2-weighted sequences $(2,5,6)$. Intramedullary involvement is quite uncommon, although surrounding soft-tissue edema may occasionally be observed (5). Periosteal chondroma demonstrates predominantly peripheral enhancement following contrast agent administration (6). In our case, the tumor was $<3 \mathrm{~cm}$ and arose on the surface of the distal tibia. The imaging characteristics were consistent with those described in the literature.

The differential diagnosis of the present case included myositis ossificans, bizarre parosteal osteochondromatous proliferation (BPOP) and periosteal chondrosarcoma. Myositis ossificans is the most common benign bone-forming lesion that mainly affects active adolescents and young adults, with a marginal male predominance (7). The lesion is associated with a single traumatic event or repeated minor trauma in the majority of the cases. The zoning phenomenon of peripheral maturation is the most significant diagnostic feature (8). BPOP, also referred to as Nora's lesion, is a surface-based osteocartilaginous lesion that typically affects the hands and feet in young adults (9) and may also occur in long bones in $\sim 25 \%$ of the cases (10). Antecedent trauma may be considered as an etiologic factor. Radiographically, BPOP typically appears as a well-marginated mass of heterotopic mineralization arising from the cortical surface without affecting the underlying bone architecture (10). The MRI characteristics are non-specific, demonstrating intermediate signal intensity on T1-weighted sequences and intermediate to high signal intensity on T2-weighted sequences, with marked contrast enhancement $(10,11)$. Histologically, BPOP consists of three components in different amounts, namely cartilage, bone and fibrous tissue. The matrix in cartilage and bone has a characteristic blue tinctorial quality at the osteocartilaginous interfaces (12). Periosteal chondrosarcoma predominantly occurs in the metaphyses of long bones and has a peak incidence between the second and fourth decades of life, with a marginal male predominance (13). The lesion is generally $>5 \mathrm{~cm}$ in diameter. Radiographically, periosteal chondrosarcoma is often round and displays granular or 'popcorn' cartilaginous opacities (14). On MRI, the mass is well-delineated, with low to intermediate signal intensity on T1-weighted sequences 
and high signal intensity on T2-weighted sequences. There is peripheral and septal enhancement following contrast agent administration (14). According to Robinson et al (15), lesion size is the most reliable predictor for distinguishing periosteal chondrosarcoma from periosteal chondroma.

In summary, we described the clinicopathological and radiological characteristics of a periosteal chondroma involving the distal tibia in a young female patient. Although rare, periosteal chondroma should be considered in the differential diagnosis of a surface-based lesion with matrix mineralization in the metaphysis of long bones.

\section{References}

1. Lucas DR and Bridge JA: Chondromas: enchondroma, periosteal chondroma. In: WHO Classification of Tumours of Soft Tissue and Bone. Fletcher CDM, Bridge JA, Hogendoorn PCW and Mertens F (eds). Vol 5. 4th edition. IARC Press, Lyon, pp252-254, 2013.

2. Kosaka H, Nishio J, Matsunaga T, Aoki M, Iwasaki H and Naito M: Imaging features of periosteal chondroma manifesting as a subcutaneous mass in the index finger. Case Rep Orthop 2014: 763480, 2014

3. Domson GF, Bush CH, Reith JR, Rajaram A, Scarborough MT and Gibbs CP: Periosteal chondroma at birth. Skeletal Radiol 37: 559-562, 2008

4. Amary MF, Bacsi K, Maggiani F, et al: IDH1 and IDH2 mutations are frequent events in central chondrosarcoma and central and periosteal chondromas but not in other mesenchymal tumours. J Pathol 224: 334-343, 2011.

5. Miller FS: Imaging features of juxtacortical chondroma in children. Pediatr Radiol 44: 56-63, 2014.
6. Woertler K, Blasius S, Brinkschmidt C, Hillmann A, Link TM and Heindel W: Periosteal chondroma: MR characteristics. J Comput Assist Tomogr 25: 425-430, 2001.

7. Rosenberg AE and Oliveira AM: Myositis ossificans and fibro-osseous pseudotumour of digits. In: WHO Classification of Tumours of Soft Tissue and Bone. Fletcher CDM, Bridge JA, Hogendoorn PCW and Mertens F (eds). Vol 5. 4th edition. IARC Press, Lyon, pp50-51, 2013.

8. Tyler P and Saifuddin A: The imaging of myositis ossificans. Semin Musculoskelet Radiol 14: 201-216, 2010

9. Berber O, Dawson-Bowling S, Jalgaonkar A, Miles J, Pollock RC, Skinner JA, Aston WJ and Briggs TW: Bizarre parosteal osteochondromatous proliferation of bone: clinical management of a series of 22 cases. J Bone Joint Surg Br 93: 1118-1121, 2011.

10. Dhondt E, Oudenhoven L, Khan S, Kroon HM, Hogendoorn PC, Nieborg A, Bloem JL and De Schepper A: Nora's lesion, a distinct radiological entity? Skeletal Radiol 35: 497-502, 2006.

11. Torreggiani WC, Munk PL, Al-Ismail K, O'Connell JX, Nicolaou S, Lee MJ and Masri BA: MR imaging features of bizarre parosteal osteochondromatous proliferation of bone (Nora's lesion). Eur J Radiol 40: 224-231, 2001.

12. Abramovici L and Steiner GC: Bizarre parosteal osteochondromatous proliferation (Nora's lesion): a retrospective study of 12 cases, 2 arising in long bones. Hum Pathol 33: 1205-1210, 2002.

13. Goedhart LM, Ploegmakers JJ, Kroon HM, Zwartkruis EC and Jutte PC: The presentation, treatment and outcome of periosteal chondrosarcoma in the Netherlands. Bone Joint J 96-B: 823-828, 2014.

14. Chaabane S, Bouaziz MC, Drissi C, Abid L and Ladeb MF: Periosteal chondrosarcoma. AJR Am J Roentgenol 192: W1-W6, 2009.

15. Robinson P, White LM, Sundaram M, Kandel R, Wunder J, McDonald DJ, Janney C and Bell RS: Periosteal chondroid tumors: radiologic evaluation with pathologic correlation. AJR Am J Roentgenol 177: 1183-1188, 2001. 